\title{
Temperature estimation from hydroxyl airglow emission in the Venus night side mesosphere
}

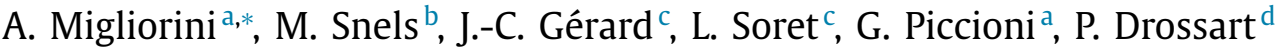 \\ a Institute of Space Astrophysics and Planetology, IAPS-INAF, Rome, Italy \\ ${ }^{\mathrm{b}}$ ISAC-CNR, Roma, Italy \\ ${ }^{\mathrm{c}} \mathrm{LPAP}$, Université de Liège, Liège, Belgium \\ ${ }^{\mathrm{d}}$ LESIA, Observatoire de Paris, PSL Research University, CNRS, Sorbonne Université, UPMC Univ. Paris 06, Univ. Paris-Diderot, Sorbonne Paris Cité, 5 place \\ Jules Janssen, 92195 Meudon, France
}

\section{A R T I C L E I N F O}

\section{Article history:}

Received 24 May 2017

Revised 11 September 2017

Accepted 21 September 2017

Available online 22 September 2017

\section{Keywords:}

Venus, atmosphere

Atmospheres, structure

Spectroscopy

Infrared observations

\begin{abstract}
A B S T R A C T
The temperature of the night side of Venus at about $95 \mathrm{~km}$ has been determined by using spectral features of the hydroxyl airglow emission around $3 \mu \mathrm{m}$, recorded from July 2006 to July 2008 by VIRTIS onboard Venus Express. The retrieved temperatures vary from 145.5 to about $198.1 \mathrm{~K}$ with an average value of $176.3 \pm 14.3 \mathrm{~K}$ and are in good agreement with previous ground-based and space observations. The variability with respect to latitude and local time has been studied, showing a minimum of temperature at equatorial latitudes, while temperature values increase toward mid latitudes with a local maximum at about $35^{\circ} \mathrm{N}$. The present work provides an independent contribution to the temperature estimation in the transition region between the Venus upper mesosphere and the lower thermosphere, by using the $\mathrm{OH}$ emission as a thermometer, following the technique previously applied to the high-resolution $\mathrm{O}_{2}\left(\mathrm{a}^{1} \Delta_{\mathrm{g}}\right)$ airglow emissions observed from ground.
\end{abstract}

(c) 2017 Elsevier Inc. All rights reserved.

\section{Introduction}

The study of Venus' atmosphere has progressed considerably in the last decade, thanks to the Venus Express space mission, which has been orbiting the planet from April 2006 to January 2015. Several experiments on board Venus Express have been used to derive the atmospheric temperature, by using either direct measurements (SOIR, VeRa, SPICAV), or through inversion methods (VIRTIS). Considering all the available datasets, the observed thermal profiles cover the altitude range from roughly $40 \mathrm{~km}$ to $150 \mathrm{~km}$, the different datasets covering overlapping ranges. A comprehensive review of the available temperature measurements and airglow observations is provided in Limaye et al. (2017) and Gérard et al. (2017), respectively. Here, we summarize the basic observations concerning the thermal structure at $90-100 \mathrm{~km}$, from space and groundbased measurements.

The atmospheric region between 90 and $100 \mathrm{~km}$ appears to be a permanent warm layer in the Venus Express/SPICAV measurements (Bertaux et al., 2007; Piccialli et al., 2015), with temperatures reaching values as high as $250 \mathrm{~K}$ at $90 \mathrm{~km}$ at the night side of Venus, with a variability with respect to latitude and local

\footnotetext{
* Corresponding author.

E-mail address: alessandra.migliorini@iaps.inaf.it (A. Migliorini).
}

time on the order of $50 \mathrm{~K}$ within the altitude range. Although this observation is in agreement with the Venus Thermospheric General Circulation Model (VTGCM) simulations (Brecht et al., 2011), which explain this warm layer with an adiabatic compression due to air subsidence on the Venus night side, the observed temperatures at $90-100 \mathrm{~km}$ are about $30 \mathrm{~K}$ warmer, compared to models reported in Zasova et al. (2006, 2007). The observing geometry of Venus Express/SOIR (Mahieux et al., 2015) limits the observations of the mesosphere and lower thermosphere $(70-150 \mathrm{~km})$ to the morning and evening terminators. The temperature measured with this instrument on board Venus Express shows a maximum value of $204 \pm 17 \mathrm{~K}$ at $0.01 \mathrm{mbar}$ (about $102 \mathrm{~km}$ height), which is reproduced by the VTGCM model (Bougher et al., 2015). Radio science measurements, obtained with the Venus Express/VeRa experiment, report values around $170 \mathrm{~K}$ at $90 \mathrm{~km}$ at all latitudes on the Venus night side (Tellmann et al., 2009). The altitude region 90$100 \mathrm{~km}$ marks the transition between the two dynamic regimes acting in the Venus atmosphere; hence turbulence and wavy structures are expected (Bougher et al., 2015). To better constraint the Global Circulation models developed to understand the Venus atmosphere, more information about the temperature at the studied altitudes is required. The recent discovery of $\mathrm{OH}$ Meinel band emission in the Venus atmosphere by Venus Express allows one to investigate the photochemistry in the upper mesosphere of the planet (Piccioni et al., 2008). The (1-0), (2-1), (3-2) and (4-3) $\mathrm{OH}$ 
transitions around $3 \mu \mathrm{m}$, as well as the (2-0) band at $1.46 \mu \mathrm{m}$ have been identified in the spectra acquired with the Visible and InfraRed Thermal Imaging Spectrometer (VIRTIS) on board the spacecraft (Piccioni et al., 2008; Migliorini et al., 2011; Soret et al., 2012). These emissions occur at about $95 \mathrm{~km}$ height (Piccioni et al., 2008; Gérard et al., 2010) and have been observed so far only on the night side of the planet. The emission altitude is somewhat variable, for example with latitude or distance from the anti-solar point. However, it was pointed out that when $\mathrm{O}_{2}\left(\mathrm{a}^{1} \Delta_{\mathrm{g}}\right)$ and $\mathrm{OH}$ emissions are observed simultaneously, the difference of their peak altitude is only about $0.5 \mathrm{~km}$ (Gérard et al., 2012). In the present study, we adopt an $\mathrm{OH}$ peak altitude emission along the line of sight of $95.3 \pm 3 \mathrm{~km}$ (Gérard et al., 2010), in comparison with the mean $\mathrm{O}_{2}$ nightglow emissions at about $95.0 \pm 2.4 \mathrm{~km}$ height, observed along the line of sight (Piccioni et al., 2009). The dominant excitation process is believed to be the $\mathrm{O}_{3}+\mathrm{H} \rightarrow \mathrm{OH}(\mathrm{v})+\mathrm{O}_{2}$ reaction yielding the hydroxyl molecule in a vibrationally excited state, followed by radiative and collisional relaxation.

The complex structure of the $\mathrm{OH}(\Delta v=1)$ band sequence at 2.8$3.2 \mu \mathrm{m}$ can be used to derive the rotational temperature at these altitudes, under the assumption of an atmosphere in local thermodynamic equilibrium (LTE). A local thermodynamic equilibrium exists when all internal degrees of freedom of a molecule are in equilibrium with the kinetic energy. This equilibrium is generally maintained through collisional transfer of energy. However, at low densities, the collision frequency is too small to guarantee an efficient transfer from the excited electronic, vibrational and rotational states to the kinetic energy of the molecule. The rotational degree of freedom is expected to be in LTE (see Rodgers et al., 1992) and thus the intensity distribution of the rotational levels can be used to estimate the temperature. However, on the Earth, it was pointed out that there is a variation of up to $15 \mathrm{~K}$ of the rotational temperature in the $v=3$ manifold with respect to the $v=8$ manifold, while the temperature difference is at most $5 \mathrm{~K}$ for the vibrational levels $v=3,4,5,6$ (Noll et al., 2015; Cosby and Slanger, 2007). In our approach, by fitting the rotational manifolds for the vibrational levels $v=1,2,3$ and 4 , we consider an average rotational temperature of the 4 vibrational levels.

The present investigation uses the rotational structure of the $\mathrm{OH}$ emission spectra recorded by the VIRTIS spectrometer aboard Venus Express to determine the temperature at the airglow altitudes $(90-100 \mathrm{~km})$. Although the spectral resolution of the VIRTIS multispectral imager is not sufficient to resolve individual rotational lines, comparison of the observed spectra with synthetic spectra convolved with the line spread function provides reliable estimates of the ambient atmospheric temperature. In the following, we describe the method and the data used to determine the rotational temperature (Section 2); results are discussed in Section 3, while a summary is provided in Section 4.

\section{VIRTIS-M data and method}

For the proposed analysis, we selected VIRTIS data acquired in the limb mode, during the period from 2006-07-06 to 2008-07-05. A detailed description of the instrument and the observing geometry can be found in Piccioni et al. (2007). The advantage of observations in limb-mode is the long path length, which provides the intensity required for detecting the low intensity $\mathrm{OH}$ emission as well as a good vertical resolution (1-2 km for a slant distance of $7000 \mathrm{~km}$ ). In addition, the full wavelength range is acquired simultaneously, as a consequence of the instrument design, and hence the measured radiance at the covered wavelengths is directly comparable. However, the necessity to use only limb observations limits the spatial coverage. The list of selected data is reported in Table 1.
Table 1

Details of the analyzed VIRTIS data. Geometric parameters are also provided.

\begin{tabular}{llll}
\hline Cube & Start time & Latitude & Local time \\
\hline VI0076-18 & $2006-07-06 T 01: 34: 11.885$ & $30.0-65.0^{\circ} \mathrm{N}$ & $21.8-23.9$ \\
VI0317-06 & $2007-03-04 T 06: 12: 02.447$ & $15.0-30.0^{\circ} \mathrm{N}$ & $23.8-0.7$ \\
VI0322-06 & $2007-03-09 T 06: 07: 58.525$ & $15.0-30.0^{\circ} \mathrm{N}$ & $0.4-1.3$ \\
VI0324-06 & $2007-03-11 \mathrm{~T} 06: 06: 22.483$ & $15.0-30.0^{\circ} \mathrm{N}$ & $0.7-1.5$ \\
VI0371-10 & $2007-04-27 \mathrm{~T} 05: 42: 00.927$ & $35.0-60.0^{\circ} \mathrm{N}$ & $21.9-23.5$ \\
VI0383-12 & $2007-05-09 T 05: 51: 47.236$ & $35.0-50.0^{\circ} \mathrm{N}$ & $18.0-0.8$ \\
VI0715-02 & $2008-04-05 T 04: 23: 08.144$ & $5^{\circ} \mathrm{S}^{\circ}{ }^{\circ} \mathrm{N}$ & $22.5-23.3$ \\
VI0718-03 & $2008-04-08 T 04: 36: 17.693$ & $0.0-20.0^{\circ} \mathrm{N}$ & $22.4-0.3$ \\
VI0724-02 & $2008-04-14 T 04: 30: 38.081$ & $0.0-5.0^{\circ} \mathrm{N}$ & $22.4-0.8$ \\
VI0792-02 & $2008-06-21 \mathrm{~T} 03: 50: 10.343$ & $45.0-70.0^{\circ} \mathrm{N}$ & $2.3-4.7$ \\
VI0806-02 $^{2008-07-05 T 04: 08: 48.562}$ & $45.0-70.0^{\circ} \mathrm{N}$ & $19.5-21.8$ \\
\hline
\end{tabular}

Data have been averaged over the $90-100 \mathrm{~km}$ altitude range, in order to increase the signal to noise ratio (SNR). Typical SNRs range from 60 to 300. Since the baseline of the observed spectra is not completely flat, a continuum subtraction was necessary prior to comparison with simulated spectra and analysis. Three different methods for baseline subtraction have been applied, in order to estimate the sensitivity of the procedure. The three different baselines have been obtained by fitting a first, second and third order polynomial, as follows:

(1) Straight line between 2.65 and $3.3 \mu \mathrm{m}$;

(2) Parabolic fit through three points: 2.6, 3.0 and $3.6 \mu \mathrm{m}$;

(3) Third order polynomial through four points: 2.0, 2.6, 3.3, $3.6 \mu \mathrm{m}$.

The points were chosen at wavelength values where no $\mathrm{OH}$ emission is observed. The emission values at the wavelengths used for the background subtraction are average values on 5-7 adjacent points. An example of the continuum estimation is shown in Fig. 1(left).

Synthetic $\mathrm{OH}$ spectra, including the contributions from the (1-0), (2-1), (3-2) and (4-3) bands, have been calculated by using the PGOPHER program (available at the following website: pgopher.chm.bris.ac.uk/) for 17 temperatures from 130 to $250 \mathrm{~K}$, and convolved with the experimental line spread function $(\mathrm{FWHM}=18 \mathrm{~nm})$ following earlier analyses and also for consistency with previous works (Soret et al., 2012). For each of the rotational temperatures, the intensities of the rotational manifolds have been fitted in the range $2.64-3.29 \mu \mathrm{m}$, in order to obtain the best agreement with observations, thus providing the relative intensity of the different vibrational transitions. Higher vibrational levels in this band, with $v \geq 4$, were ignored since their spectral features were too noisy and not unambiguously identified. Successively, a chisquared test has been performed to determine which rotational temperature provided the best fit, by fitting a third order polynomial through the chi-squared values obtained for each temperature. The fit for VIRTIS image VI0715-02 is shown in Fig. 1. In this case, the minimum $\chi^{2}$ value is obtained for $T_{\text {rot }}=186.3 \pm 1.1 \mathrm{~K}$. A final value for the temperature has been obtained by averaging the values resulting from the three fits with a different continuum subtraction.

The statistical error in the determination of the temperature depends both on the baseline subtraction and on the SNR of the averaged spectra, as well as on other sources of error that are difficult to quantify. Here we estimate the error produced by the baseline subtraction as the standard deviation of the three fits with different baseline subtraction. The error due to the sensor noise has been estimated by using synthetic spectra with added noise for each spectrum taking into account the noise equivalent spectral radiance (NESR) of the detector and the number of observations averaged for the specific spectrum. The statistical variation in the 

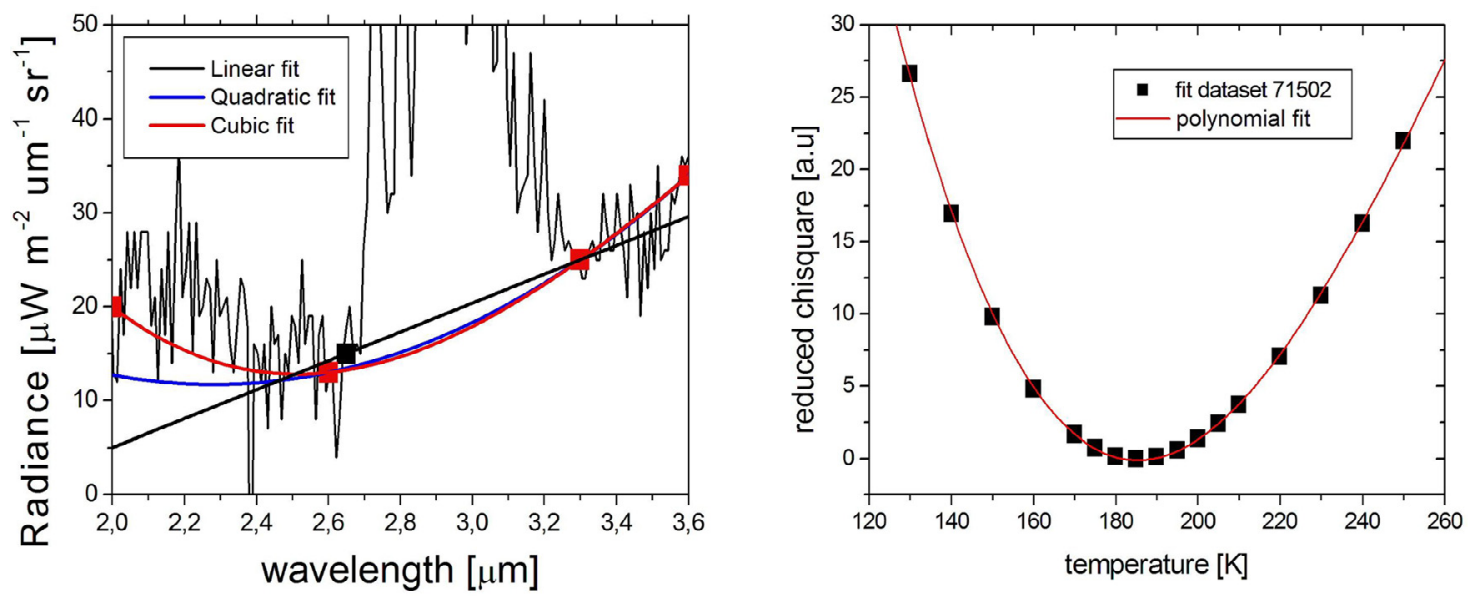

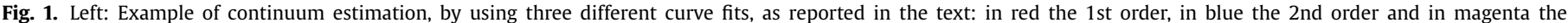

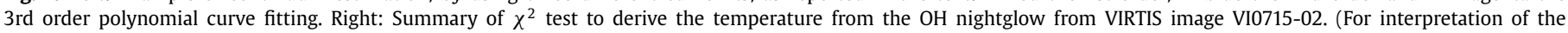
references to colour in this figure legend, the reader is referred to the web version of this article.)

Table 2

Temperature estimation for each image. Temperature values are reported in column 2, the number of spectra per image in column 3 , the estimated noise, noise maximum and signal-to-noise ratio in columns 4, 5, 6 respectively, errors on noise and baseline in columns 7 and 8 , and total estimated error in column 9.

\begin{tabular}{|c|c|c|c|c|c|c|c|c|}
\hline Image & Temp (K) & $\mathrm{N}$ spectra & Noise $10^{-4}$ & Max $10^{-4}$ & $\mathrm{~S} / \mathrm{N} \max$ & Error noise (K) & Error baseline (K) & Error total $(\mathrm{K})$ \\
\hline $317-06$ & 172.6 & 584 & 0.2 & 60 & 300 & 0.9 & 1 & 1.3 \\
\hline $371-10$ & 184.1 & 576 & 0.21 & 56 & 267 & 1 & 3.1 & 3.3 \\
\hline $383-12$ & 198.1 & 819 & 0.17 & 14 & 82 & 3.2 & 5.8 & 6.6 \\
\hline $715-02$ & 186.3 & 5906 & 0.07 & 21 & 300 & 0.9 & 0.7 & 1.1 \\
\hline 792-02 & 178 & 673 & 0.19 & 7.5 & 40 & 6.3 & 9.5 & 11.4 \\
\hline 806-02 & 166.2 & 604 & 0.2 & 13 & 65 & 3.9 & 2.2 & 4.5 \\
\hline
\end{tabular}

temperature with respect to the amount of added random noise was obtained by repeating the fit 500 times, each time generating an independent random noise with the same root-mean-square value. The standard deviation in these fits provided the error in the rotational temperature due to the noise. The total statistical error in the determination of the rotational temperature is estimated from these two contributions, assuming that they can be considered independent. Table 2 summarizes the fits and the determination of the statistical errors for the analyzed VIRTIS images. Image VI0322-06 was excluded from the investigation because, although temperature estimation can be obtained from this measurement, the value is unphysically high and the calculated errors very large compared to the rest of the dataset. This could be attributed to the bad quality of the measurement, with a consequent unfavorable SNR.

We also explored the influence of the chosen instrumental line width on the determination of the rotational temperatures, by repeating the procedure described above starting with synthetic spectra convolved at a Full Width at Half Maximum (FWHM) of 16 and $20 \mathrm{~nm}$. A significant correlation between the obtained temperature and the chosen instrumental FWHM was evident. For an increment of $1 \mathrm{~nm}$ in the linewidth, an average decrease of $3 \mathrm{~K}$ is observed. Assuming that the proper instrumental linewidth is known within $\pm 1 \mathrm{~nm}$, this implies an error of $\pm 3 \mathrm{~K}$ in the temperature. This error, however, is not a statistical, but a systematic error; a different choice of the instrumental linewidth shifts the temperature of all spectra in the same way. This analysis has been applied to 10 VIRTIS images, which probe different regions of the Venus' night-side atmosphere in terms of latitude and local time, as reported in Table 1. Possible temperature variations with latitude and local time have been investigated, and results are discussed in the following section.

\section{Results}

Fig. 2 shows examples of the simulated spectra providing the best fits to the observed spectra 317-06, 371-10 and 715-02. The minimum chi-square is obtained for the fit with $T_{\text {rot }}=172.6,184.1$, and $186.3 \mathrm{~K}$, respectively. Following the procedure described above, 10 different observations have been analyzed, covering several latitudes, in the northern hemisphere, and local times.

The obtained temperature values, which are reported in Table 2, show a minimum of $145.5 \pm 2.2 \mathrm{~K}$ for image VI0718-03, which probes the latitude range $0-20^{\circ} \mathrm{N}$, and a maximum of $198.1 \pm 6.6 \mathrm{~K}$ at latitudes $35-50^{\circ} \mathrm{N}$ (VIRTIS image VI0383-12). The mean temperature is of $176.3 \pm 14.3$, in good agreement with temperature estimations obtained by using ground-based measurements of the high-resolution $\mathrm{O}_{2}\left({ }^{1} \Delta\right)$ emission observations (Connes et al., 1979; Crisp et al., 1996; Ohtsuki et al., 2008; Bailey et al., 2008; Krasnopolsky, 2010). In this case, it is assumed that the rotational temperature is equal to the air temperature because of the local thermodynamic equilibrium condition, which is satisfied by the excited $\mathrm{O}_{2}$ molecules that have a sufficiently long lifetime to thermalize the layer. By combining these high-resolution observations of $\mathrm{O}_{2}$ airglow with the vertical profile measurements at limb with Venus Express, it was possible to infer the altitude of the airglow emission. Values reported in literature range between $171 \mathrm{~K}$ and $212 \mathrm{~K}$, depending on the latitude and the observing technique. Long term monitoring of the thermal field shows a small variation on yearly time scales, according to Ohtsuki et al. (2008). The 

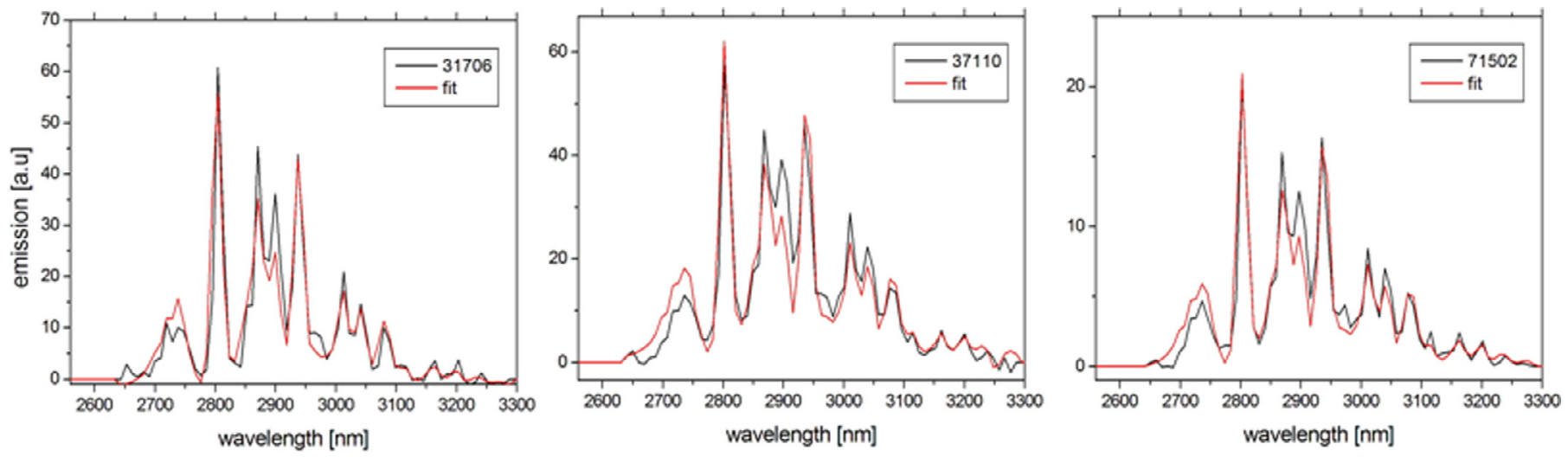

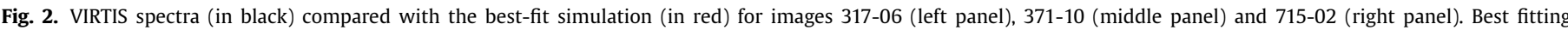

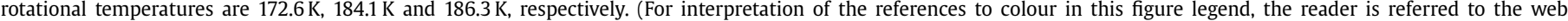
version of this article.)

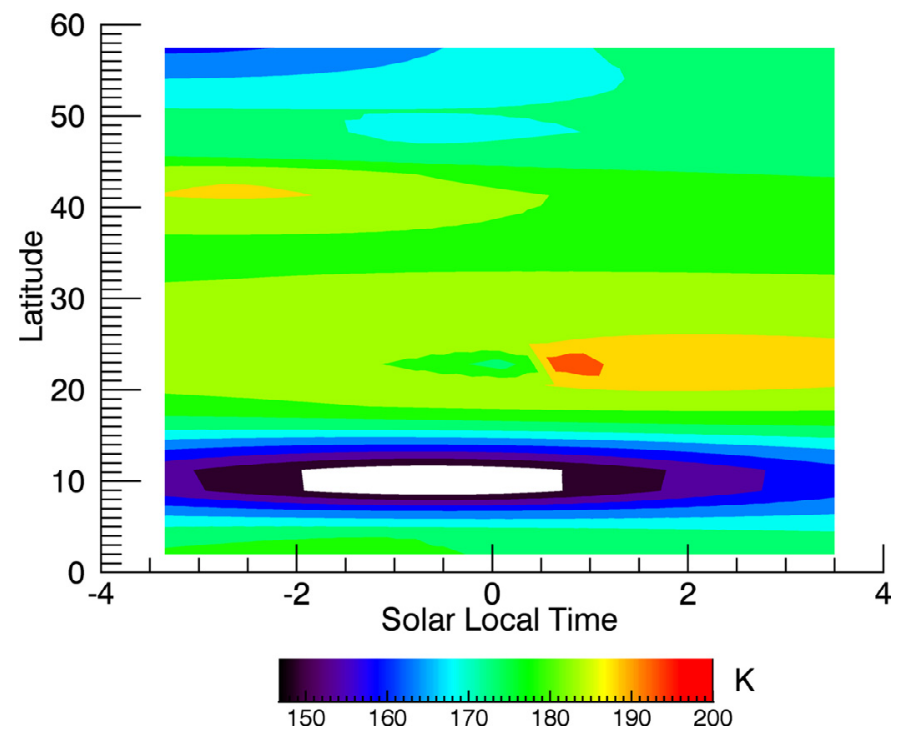

Fig. 3. Thermal field obtained with the considered 10 VIRTIS-M images. A minimum is observed close to the equatorial latitudes, while temperature increases toward mid-latitudes.

average rotational temperatures, during different observing ground based campaigns from 2002 to 2007 , vary in the range $193 \pm 9 \mathrm{~K}$ in the hot region observed in the anti-solar point, $182 \pm 25 \mathrm{~K}$ at mid-latitudes a few hours after midnight and $185 \pm 20 \mathrm{~K}$ at mid latitudes in the dusk hemisphere within a few hours from the anti-solar point. Variability of $20-30 \mathrm{~K}$ with latitude is reported in Krasnopolsky (2010), as will be discussed below.

We now consider how the temperature varies with respect to latitude and local time. Fig. 3 provides the variability of estimated temperature with local time and latitude. Despite the limited number of analyzed images, some preliminary results can be highlighted. A local minimum of temperature is observed at 5$20^{\circ} \mathrm{N}$ at all the local times covered by the selected VIRTIS images, while temperature increases in the latitude range $20-30^{\circ} \mathrm{N}$. However, considering the limited number of analyzed images, it is quite difficult to draw firm conclusions. Krasnopolsky (2010) reported a local minimum of $171 \mathrm{~K}$ at equatorial latitudes $\left(4^{\circ} \mathrm{S}\right)$, with an increase of the temperature to $195 \mathrm{~K}$ at $35^{\circ} \mathrm{S}$ and $212 \mathrm{~K}$ at $35^{\circ} \mathrm{N}$, with uncertainty of $5 \mathrm{~K}$, as deduced from high resolution ground-based measurements. The same trend is observed in our map, although our values at mid northern latitudes are substantially lower than those reported in Krasnopolsky (2010).
A comparison with previous measurements is provided in Fig. 4. The temperature estimation presented in this work is in agreement with the VeRa mean profile, and in partial accord with the previous $\mathrm{O}_{2}$ ground-based measurements. However, the temperature from the $\mathrm{OH}$ airglow is about $8 \mathrm{~K}$ higher than the VIRA profile for northern latitudes (Seiff, 1983). Although a temperature of about $220 \mathrm{~K}$ is detected in the case of VIRTIS image VI0322-06, our measurements are always $30-40 \mathrm{~K}$ lower than the SPICAV temperature values at $95 \mathrm{~km}$.

Since previous works suggest that there should be a considerable temperature gradient between 90 and $100 \mathrm{~km}$ (Bertaux et al., 2007; Piccialli et al., 2015; Mahieux et al., 2015), we tried to refine our procedure by fitting layers of $3 \mathrm{~km}$ separately and determine the rotational temperature for each layer. Despite the larger statistical errors in this case than when the full $90-100 \mathrm{~km}$ layer is considered, a trend might be observed. Results are shown in Fig. 5 for seven images. The statistical errors have been calculated from the standard deviation of the temperatures determined from three different baseline subtractions and from the SNR for each layer. Except for the VI0806-02 image, which shows a much lower temperature in all the layers, other images exhibit temperatures between 160 and $195 \mathrm{~K}$. Temperatures seem to be generally lower in the top layer $(100 \mathrm{~km})$, except for image VI0715-02. A possible local maximum at about $97 \mathrm{~km}$ is present in the VI0324-06, VI0371-10 and VI0383-12 images, while no clear trends are evident in the other cases. These spectra were collected in the latitude region between $15^{\circ} \mathrm{N}$ and $60^{\circ} \mathrm{N}$, around midnight. However it is difficult to derive a clear trend, due to the limited dataset and the observed high variability.

It might be noticed, however that the considered images are probing different latitudes and local times, implying that the temperatures determined from the fits of $2-3 \mathrm{~km}$ layers cannot be directly compared. Moreover, the $90-100 \mathrm{~km}$ altitude region is known to be highly variable, as shown also in the analysis of SPICAV (Bertaux et al., 2007; Piccialli et al., 2015) and SOIR data (Mahieux et al., 2015). Hence deviations from the general trend might reflect a real variability of the atmosphere.

\section{Conclusions}

The $\mathrm{OH}(\Delta v=1)$ Meinel sequence, at 2.6-3.3 $\mu \mathrm{m}$ has been studied by using VIRTIS/Venus Express data. Eleven limb images in this spectral region have been analyzed to determine the atmospheric temperature between 90 and $100 \mathrm{~km}$, where the $\mathrm{OH}$ airglow emission peak has been observed (Piccioni et al., 2008). Temperature measurements at these altitudes are quite debated. A permanent warm layer was reported in the thermal structure derived from 

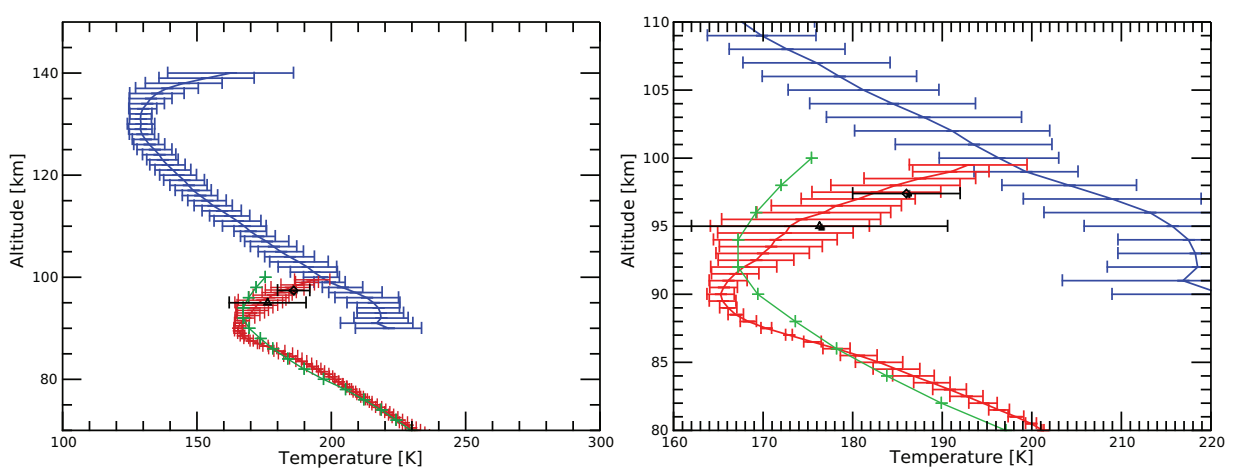

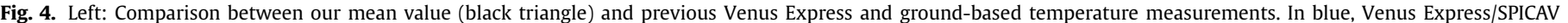

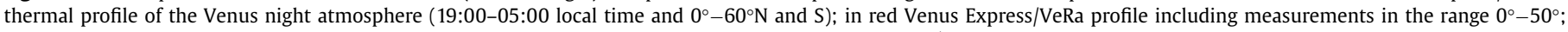

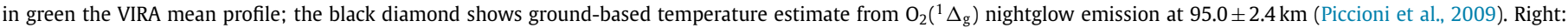

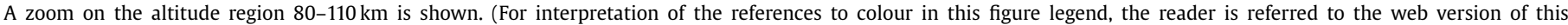
article.)

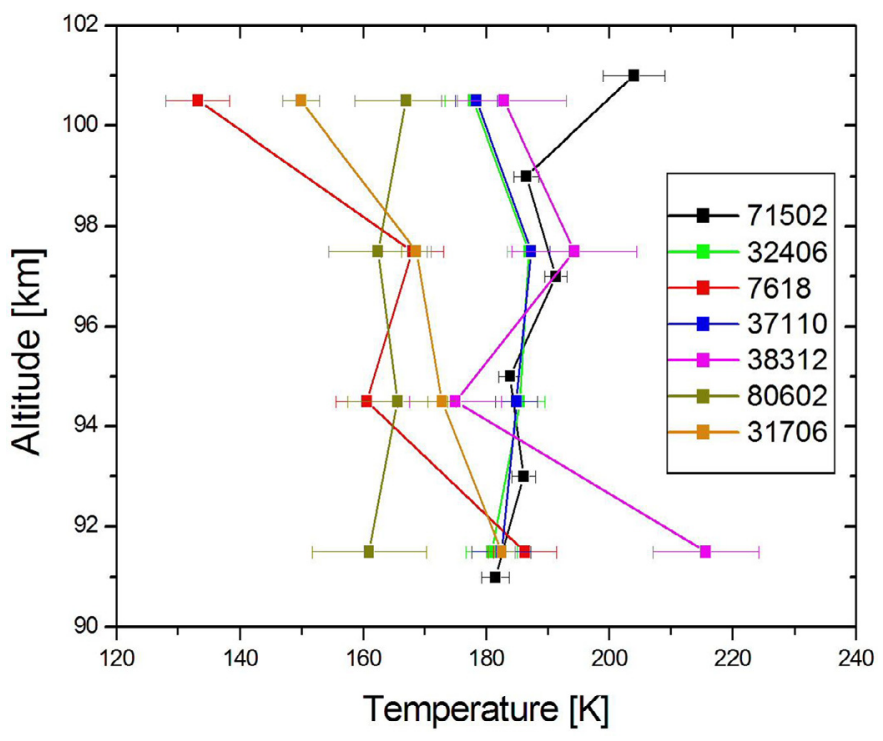

Fig. 5. Temperature variability with altitude in height bins $3 \mathrm{~km}$ wide.

the SPICAV/Venus Express data in the altitude region $90-100 \mathrm{~km}$, with temperatures as high as $250 \mathrm{~K}$ (Piccialli et al., 2015), while the SOIR solar occultation technique reveals temperatures between 171 and $204 \mathrm{~K}$ at 87 and $102 \mathrm{~km}$ respectively at the morning terminator (Mahieux et al., 2015). Previous ground-based observations used the rotational structure of the $\mathrm{O}_{2}\left({ }^{1} \Delta_{\mathrm{g}}\right)$ nightglow emission (Connes et al., 1979; Crisp et al., 1996; Ohtsuki et al., 2008; Bailey et al., 2008; Krasnopolsky, 2010) to determine the rotational temperature. Values obtained from $\mathrm{O}_{2}$ nightglow reported in literature range from $171 \mathrm{~K}$ up to $212 \mathrm{~K}$, depending on the latitude and the observing technique. Here we have used VIRTIS spectra acquired from July 2006 to July 2008, to derive rotational temperatures ranging from $145 \mathrm{~K}$ to $200 \mathrm{~K}$, with a statistical error in the temperature retrieval of about $4 \mathrm{~K}$ and a systematical error of $\pm 3 \mathrm{~K}$ depending on the choice of the width of the instrumental line spread function. The average temperature value obtained with this technique is $176.3 \pm 14.3 \mathrm{~K}$, in fairly good agreement with the ground-based measurements listed above. Variability with latitude and local time suggests the presence of a local minimum at equatorial latitudes, and an increase of temperature at mid latitudes around midnight, although the too poor sample has not a statistical meaning. The applied method is an effective alternative to derive temperatures at an altitude not well investigated with other remote sensing or occultation measurements with the instruments onboard Venus Express. Hence, the present work contributes with independent data to the temperatures estimation of the upper mesosphere-lower thermosphere of Venus, providing new constraints on the aeronomy of Venus. We finally note that the analysis discussed in the present paper is based on our best knowledge of $\mathrm{OH}$ characteristics in an Earth-like atmosphere, i.e. dominated by $\mathrm{O}_{2}$ and $\mathrm{N}_{2}$. The Venus atmosphere is mainly composed of $\mathrm{CO}_{2}$, but the behavior of $\mathrm{OH}$ in such atmospheric composition and conditions is still too poorly investigated. New laboratory experiments on hydroxyl in an atmosphere dominated by $\mathrm{CO}_{2}$ are highly desirable to address this point.

\section{Acknowledgments}

The authors thank ESA, ASI and the other national space agencies, which supported the Venus Express mission. This work was funded by Italian Space Agency (ASI) (grant no. ASI-INAF I/050/10/2). JCG acknowledges support from the SCOOP-BRAIN research contract Nr. BR/143/A2/SCOOP managed by the Belgian Federal Science Policy Office (BELSPO).

\section{References}

Bailey, J., Meadows, V.S., Chamberlain, S., Crisp, D, 2008. The temperature of the Venus mesosphere from $\mathrm{O}_{2}\left(\mathrm{a} \Delta \mathrm{g}^{1}\right)$ airglow observations. Icarus 187, 247-259.

Bertaux, J.-L., Vandaele, A.-C., Korablev, O., et al., 2007. A warm layer in Venus' cryosphere and high-altitude measurements of $\mathrm{HF}, \mathrm{HCl}, \mathrm{H}_{2} \mathrm{O}$ and $\mathrm{HDO}$. Nature 450, 646-649.

Bougher, S.W., Brecht, A.S., Schulte, R., et al., 2015. Upper atmosphere temperature structure at the Venusian terminators: a comparison of SOIR and VTGCM results. Planet. Space Sci. 113-114, 336-346.

Brecht, A.S., Bougher, S.W., Gérard, J.-C., Parkinson, C.D., Rafkin, S., Foster, B., 2011. Understanding the variability of nightside temperatures, NO UV and $\mathrm{O}_{2}$ IR nightglow emissions in the Venus upper atmosphere. J. Geophys. Res. 116, E08004.

Connes, P., Noxon, J.F., Traub, W.A, et al., 1979. $\mathrm{O}_{2}\left({ }^{1} \Delta\right)$ emission in the day and night airglow of Venus. Astrophys. J. 233, L29-L32.

Cosby, P.C., Slanger, T.G., 2007. OH spectroscopy and chemistry investigated with astronomical sky spectra. Can. J. Phys. 85, 77-99.

Crisp, D., Meadows, V.S., Bezard, B., et al., 1996. Ground-based near-infrared observations of the Venus night side: near-infrared $\mathrm{O}_{2}\left(\mathrm{a}^{1} \Delta_{\mathrm{g}}\right)$ airflow from the upper atmosphere. J. Geophys. Res. 101, 4577-4594.

Gérard, J.-C., Bougher, S.W., López-Valverde, M.A., et al., 2017. Aeronomy of the Venus upper atmosphere. Icarus in press.

Gérard, J.-C., Soret, L., Piccioni, G., Drossart, P., 2012. Spatial correlation of OH Meinel and $\mathrm{O}_{2}$ infrared atmospheric nightglow emissions observed with VIRTIS-M on board Venus Express. Icarus 217, 813-817.

Gérard, J-C Soret, L., Saglam, A et al., 2010. The distribution of the $\mathrm{OH}$ Meinel and $\mathrm{O}_{2}\left(\mathrm{a}^{1} \Delta-\mathrm{X}^{3} \Sigma\right)$ nightglow emissions in the Venus mesosphere based on VIRTIS observations. Adv. Space Res. 45, 1268-1275.

Krasnopolsky, V.A., 2010. Venus night airglow: ground-based detection of OH, observations of $\mathrm{O}_{2}$ emissions, and photochemical model. Icarus 207, 17-27. 
Limaye, S.S., Lebonnois, S., Mahieux, A., et al., 2017. The thermal structure of the Venus atmosphere: intercomparison of Venus Express and ground based observations of vertical temperature and density profiles. Icarus 294, 124-155.

Mahieux, A., Vandaele, A.-C., Bougher, S.W., et al., 2015. Update of the Venus density and temperature profiles at high altitude measured by SOIR on board Venus Express. Planet. Space Sci. 113-114, 309-320.

Migliorini, A., Piccioni, G., Cardesín Moinelo, A., Drossart, P., 2011. Hydroxyl airglow on Venus in comparison with Earth. Planet. Space Sci. 59, 974-980.

Noll, S., Kausch, W, Kimeswenger, S., et al., 2015. OH populations and temperatures from simultaneous spectroscopic observations of 25 bands. Atmos. Chem. Phys. $15,3647-3669$.

Ohtsuki, S., Iwagami, N., Sagawa, H., et al., 2008. Imaging spectroscopy of the Venus 1.27- $\mu \mathrm{m} \mathrm{O}_{2}$ airglow with ground-based telescopes. Adv. Space Res. 41, $1375-1380$.

Piccialli, A., Montmessin, F., Balyaev, D., et al., 2015. Thermal structure of Venus nightside upper atmosphere measured by stellar occultations with SPICAV/Venus Express. Planet. Space Sci. 113-114, 321-335.

Piccioni, G., et al., 2007. In: ESA Special Publication, Vol. SP 1295, p. 27.

Piccioni, G., Drossart, P., Zasova, L., et al., 2008. First detection of hydroxyl in the atmosphere of Venus. A\&A 483, L29-L33.
Piccioni, G., Zasova, L., Migliorini, A., Drossart, P., Shakun, A., García-Munoz, A., Mills, F.P., Cardesin-Moinelo, A., 2009. Near-IR oxygen nightglow observed by VIRTIS in the Venus upper atmosphere. J. Geophys. Res. 114, E00B38. doi:10. 1029/2008JE003133.

Rodgers, C.D., Taylow, F.W., Muggeridge, A.H., et al., 1992. Local thermodynamical equilibrium of carbon dioxide in the upper atmosphere. Geophys. Res. Lett. 19, 589-592.

Seiff, A., 1983. Thermal structure of the atmosphere of Venus. In: Venus. University of Arizona Press, pp. 215-279.

Soret, L., Gérard, J-C. Piccioni, G., Drossart, P., 2012. The $\mathrm{OH}$ Venus nightglow spectrum: intensity and vibrational composition from VIRTIS-Venus Express observations. Planet. Space Sci. 73, 387-396.

Tellmann, S., Pätzold, M., Häusler, B., et al., 2009. Structure of the Venus neutral atmosphere as observed by the Radio Science experiment VeRa on Venus Express. J. Geophys. Res. 114, E00B36. doi:10.1029/2008JE003204.

Zasova, L.V., Ignatiev, N., Khatuntsev, I., Linkin, V., 2007. Structure of the Venus atmosphere. Planet. Space Sci. 55, 1712-1728.

Zasova, L.V., Moroz, V.I., Linkin, V.M., et al., 2006. Structure of the Venusian atmosphere from surface up to $100 \mathrm{~km}$. Cosmic Res. 44, 364-383. 